\title{
How Accurate are Patient Histories? Hepatitis B Knowledge and Prevalence among Dental Patients
}

\author{
Mohamed Yaser Kharma ${ }^{1,2 *}$, Baydaa Koussa ${ }^{3}$, Madeha Sadki ${ }^{3}$, Mohamad \\ Almech ${ }^{4}$, Sulaiman Alamari ${ }^{4}$, Jumana Yaseen ${ }^{4}$, Hala Alras ${ }^{4}$ and Ahmed \\ Aldwaik $^{4}$ \\ ${ }^{1}$ Professor in Oral Maxillofacial Surgery, Aleppo University, Faculty of Dentistry, Syria \\ ${ }^{2}$ Professor in Oral Maxillofacial Surgery, Vision Colleges, Jeddah, Saudi Arabia \\ ${ }^{3}$ Msc in Oral Maxillofacial Surgery, Vision Colleges, Jeddah, Saudi Arabia \\ ${ }^{4}$ Intern in Vision Colleges, Jeddah, Saudi Arabia \\ *Corresponding Author: Mohamed Yaser Kharma, Professor in Oral Maxillofacial \\ Surgery, Aleppo University, Faculty of Dentistry, Syria and Professor in Oral Maxil- \\ lofacial Surgery, Vision Colleges, Jeddah, Saudi Arabia.
}

Received: July 19, 2021

Published: August 19, 2021

(C) All rights are reserved by Mohamed Yaser

Kharma., et al.

\begin{abstract}
Background: The importance of adequate medical history taking in dentistry is essential and interferes with the patient's dental treatment. But the validity of such data is often questioned. Trust is the key element among doctor-patient helps to establish solid medical history, achieve better health outcomes and better perceived quality of healthcare services.

Aim: The aim of current study was to evaluate the accuracy of past medical history in dental patients and correlate the acquired information with clinical medical tests. We undertook a preliminary investigation of the validity of patient histories obtained regarding the most important diseases noticed in dental clinic (hepatitis B-Diabetes).

Materials and Methods: A self-administrated questionnaires were distributed randomly to 200 dental patients who treated in Vision colleges/Jeddah for dental problems to evaluate their knowledge, awareness and prevention methods about hepatitis, with hepatitis B and Diabetes tests to all participants. Data analyzed as statistical descriptive study.

Result: In this study, we can conclude that dental patients have a fairly unsatisfied level of knowledge and awareness regarding HBV infection. The collected data were reasonably accurate, about $2 \%$ of participants were testing positive with hepatitis, and about $5 \%$ of participants were testing diabetes which not associate with the collected data.

Conclusion: There is some degree of reliance in the past medical histories collected by the patients therefor accurate diagnosis and maximum of infection control must be applied in all patients to achieve high standard protection for the patients and the health care professional.
\end{abstract}

Keywords: Accuracy; Trust; Doctor-Patient Relationship; Hepatitis B; Diabetes

\section{Introduction}

Patient safety is an important medical discipline which aims to improving quality of patient care, minimizing treatment mistakes and enhancing safety [1].

The information collecting by a patient's past medical history will usually influence the evaluation and care of that patient.
However, this information may not always be accurate. In addition, there is a screening clinical test available and used as a marker for the validity of the patient's history [2].

The means of collecting medical data vary widely. There are three basic methods to obtain a medical history: (1) Using a printed questionnaire; (2) Verbal communication or (3) Combined [3]. Some studies assessed the reliability of a self-administered ques- 
tionnaire and significant discrepancies were revealed not only in the self-administered questionnaire but also in the history taken by the receiving healthcare worker $[4,5]$.

Some patients feel the risks associated with disclosing their status are threatening enough to make them keep this information to themselves when seeking dental care [6].

It is important to be able to ask the patient discriminating questions about particular symptoms to verify their actual nature and give enough information to support the process of reaching a diagnosis. However, we should emphasize about the degree of trust and the accuracy of what patients say about their medical history.

Trust between the patient and the healthcare provider is important between doctor-patient interaction. It influences patient management outcomes [7]. It can also be defined as a feeling of reassurance or confidence in the doctor [8].

Validity of the patient is defined as the accuracy with which a test result agrees with the truth $[9,10]$.

Hepatitis B is blood borne infection disease caused by hepatitis $B$ virus (HBV) with high risks of transmission in dental practice [11].

Saudi Ministry of Health $(\mathrm{MOH})$ revealed that viral hepatitis is considered as second most common viral infection after chickenpox. Prevalence of hepatitis is $3.2 \%$ for the Saudi Arabia which is slightly lower than the worldwide prevalence of 3.6\% [12].

Hepatitis B infection is a transmissible disease and is predominantly transmitted through blood and blood products of infected patients [13]. Transmission of infectious disease can occur easily in dental clinics [10]. Infections can be transmitted in the dental clinic through infected needles, direct blood contact, oral saliva, and indirect contact with contaminated instruments, operatory equipment, or environmental surfaces [14]. This can be prevented by the standard precaution that has been adopted by the U.S. Centers for Disease Control and Prevention (CDC) in 1996 [15]. This precaution should be applied by the dentist and dental team to overcome the risk of cross infection. HBV vaccination is obligatory requirement by the dental and medical schools in Saudi Arabia [16].

Diabetes is also another common disease worldwide. Type 1 diabetes is sometimes called insulin-dependent or juvenile diabe- tes. Type 2 diabetes is the most common form of the disease and is sometimes known as adult-onset diabetes. Prevalence of Diabetes in Saudi Arabia is currently 18.3\% [17].

Hyperglycemia can cause several complications related to different organ systems especially the eyes, kidneys, nerves, heart, and blood vessels. Also, diabetes has an adverse effect on periodontal health, and periodontal infection [18].

However, it has been found that up to $22 \%$ of individuals with diabetes also do not self-report their medical history [19].

The identification of factors that influence the accuracy of information obtained at the time of medical interviews will assist the physician in adequately assessing and treating patients.

\section{Materials and Methods}

This study was approved by Vision colleges Institutional Ethical Review Board in Jeddah. The study was conducted at the Department of Oral maxillofacial surgery via a self-administrated questionnaire with hepatitis and Diabetes tests to all participants. The data was collected from October to December 2020. In this study patients younger (less than 20 years of age) were excluded. All the participants were personally interviewed and anonymity was assured.

The questionnaires were distributed randomly to 200 dental patients who treated in Vision colleges /Jeddah for dental problems. In this study a patients under 15 years of age were excluded. The questionnaire consisted of three sections: 1) demographics and personal information, 2) evaluation of HBV knowledge and awareness of prevention 3 ) results of HBV and diabetes tests and validity of patient's medical history. Patients who agreed to participate in the study, signed a consent form prior to answer the questionnaire and perform the tests.

Serological markers like hepatitis B surface antigen (HBsAg) was usually used to evaluate the different state of the disease. With regard to serology of the disease, presence of the antigen HBsAg not only indicates active disease but can also be used to diagnose chronic carrier status. Hence, detection of HBsAg in serum is of high diagnostic value [20].

We used immunochromatography test to detect HBsAg in blood of dental patients (ABON Hepatitis B surface antigen rapid test 
device) as the technique has high sensitivity $96 \%$, specificity $98 \%$ and can be performed as a chairside test [21].

For checking the diabetes, self-monitoring of blood glucose (SMBG) using finger-stick blood samples, test strips, and portable meters has used (Accu-check glucometers: sensitivity $81 \%$ and specificity 65\%) [22]. Patient consider as diabetes if fasting test over $140 \mathrm{mg} / \mathrm{dl}$, random test over $200 \mathrm{mg} / \mathrm{dl}$ [23].

Accuracies and omissions of medically significant factors were noted and evaluated. The diagnostic tests were compared to the patient's responses and the degree of matching scored classified in two categories: (1) valid, (2) not valid. Validity is related to patient history and correlated with the result of the tests. If the patient denied the presence of hepatitis and diabetes and the tests confirmed it was assigned as not valid and where the match was exact it was assigned as valid.

\section{Results}

200 patients participated in this study, the participants comprised 116 (58\%) male and 84 (42\%) female. total of 49\% (98) were Saudi and 51\% (102) were non-Saudi.

Regarding the knowledge of participants for HBV: A $61.5 \%$ (123) of participants had no idea about hepatitis and only $20 \%$ (40) had knowledge about the signs and symptoms (Figure 1). Furthermore, only $27 \%$ (54) of participants had knowledge about the mode of transmission and the methods of prevention (Figure 2).

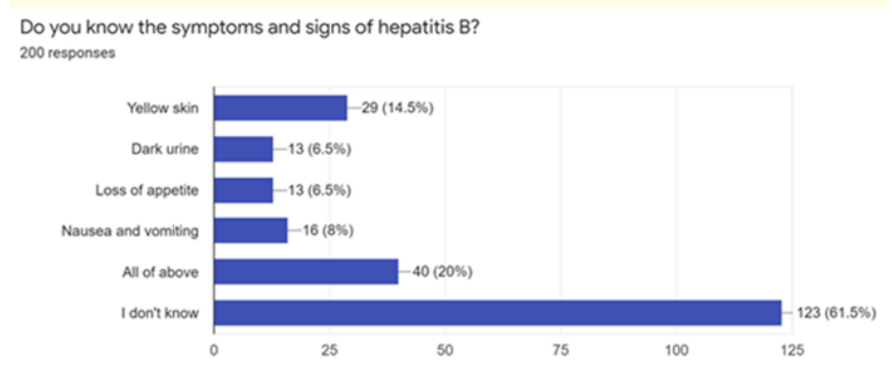

Figure 1: Knowledge of participant about signs and symptoms of hepatitis

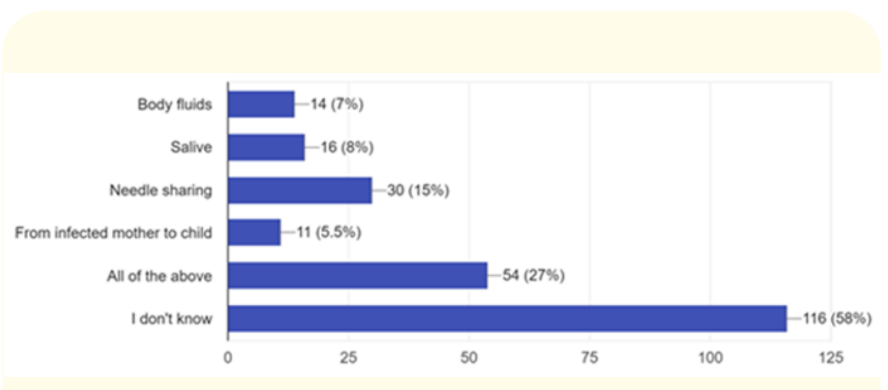

Figure 2: Knowledge of participant about mode of spreading hepatitis.

The rate of fear to get infection with hepatitis during dental treatment was about 56.5\% (113) and only 32.8\% (60) of participants were vaccinated against HBV.

A sizable proportion (59.5\%) of the respondents ignores the parameters of prevention and protection to expose to HBV. nevertheless $26 \%$ (52) had reported the correct information about the methods of prevention.

About 56.5\% (113) of the participants knew about the possibility of HBV transmission through saliva and from dentist to patient (Figure 3).

Do you believe that $\mathrm{HBV}$ infection is transmitted in dental clinics during dental treatments? 200 responses
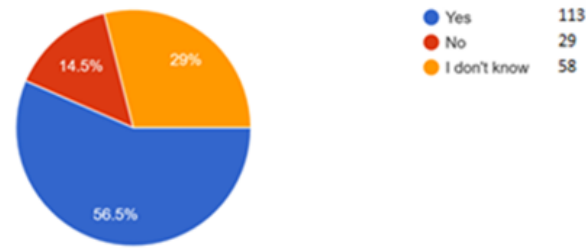

Figure 3: Perception of participant about transmission hepatitis from dentist to patient.

The validity and the accuracy of the collected past medical histories for the participants were evaluated only for HBV and Diabetes by two tests immunochromatography and glucometers respectively. 
The past medical history collected by the patients indicate that more than $91 \%$ were non diabetes and only one declaration of hepatitis was registered during collecting medical histories (Figure 4).

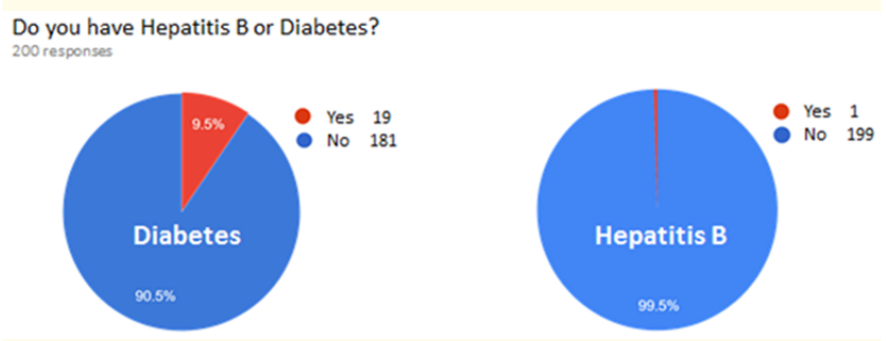

Figure 4: Recorded medical histories for hepatitis and diabetes among participants.

However, the result of glucometers support that about $4 \%$ of non-declared diabetes were diabetes (Figure 5).

Also, only one of participants had HBV according to their past medical histories. nevertheless, ABON Hepatitis B test were positive for $4(2 \%)$ of participant and the results were reconfirmed by serology test of HBV (Figure 5 and 6).

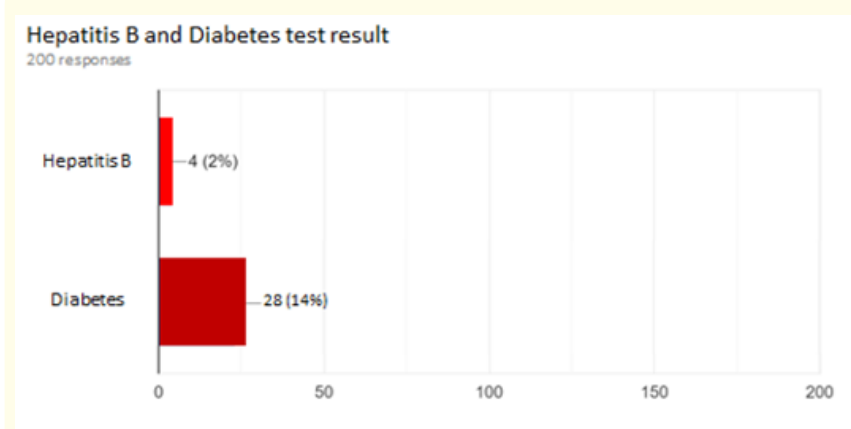

Figure 5: Hepatitis B and diabetes test results.

Over $95 \%$ of our sample showed reasonable agreement between self-reported medical histories and verified medical diseases (fairly percentage of inaccuracy of patient's histories) (Figure 7).

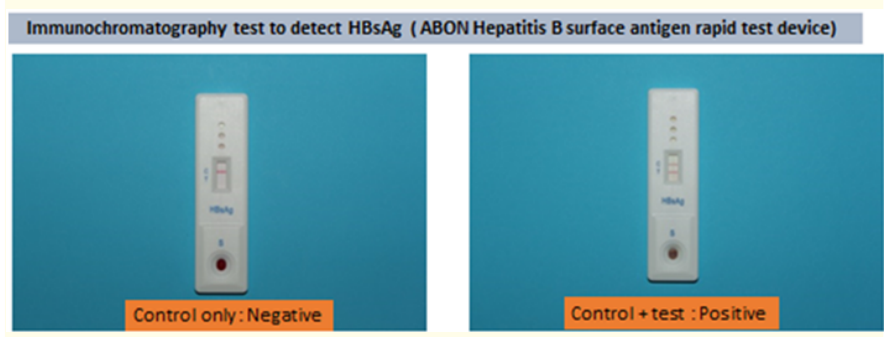

Figure 6: ABON Hepatitis B test results.

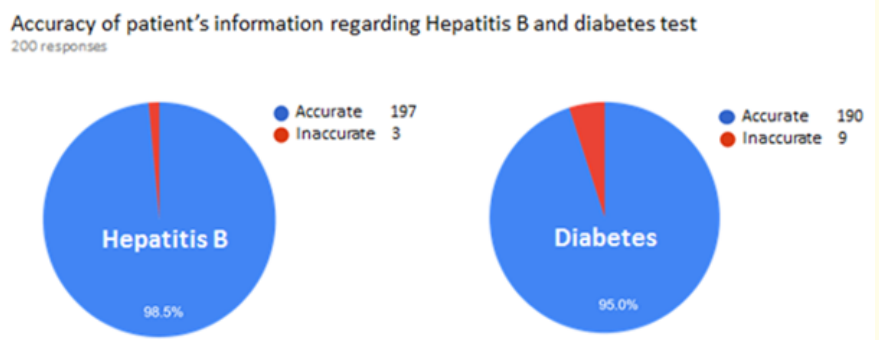

Figure 7: Accuracy of patient's information regarding hepatitis B and diabetes.

\section{Discussion}

The medical history is an important factor in physical examination. The possibility of therapeutic errors in clinical dentistry may be in somewhat due to incomplete or incorrect information in medical histories. Underestimates of some screening procedures and underreporting of medical conditions and risk behaviors have been attributed to screening examination and to the way of collecting patient's health histories [24].

Lack of knowledge regarding HBV infection among general populations can be responsible for the rapid and uncontrolled spread of HBV [25].

Based on the results of this study, four patients were found to have hepatitis B and 23 have Diabetes. we can deduce that dental patients have a fairly unsatisfied level of knowledge and awareness regarding HBV infection. Also, it highlighted the need for further HBV education for the non-medical community. 
In other word, trust and communication is considered to play a vital role in the doctor-patient relationship and the collected medical history remains a crucial part of the assessment of any patient [26]. From a clinical perspective the effective and efficient communication is a tool that helps doctors in providing quality care to their patients [27].

The presence or absence of trust between doctor-patient has practical implications. Studies have shown that trust is closely related and somewhat influenced by the health care providers' communication skills [28]. Training the medical staffs on proper interactions with the patient will contribute to increase in quality of doctor-patient interaction. Nevertheless, refusing a patient access to an additional clinical investigation may be indicator that the patient is not telling the truth about their past medical history. The hesitation of patients to participate in 2 tests (Diabetes-Hepatitis B) in the same visit could be consider as a limitation of this study.

In any way, future studies of the validity of medical interviews are recommended and should involve sufficient numbers of patients.

\section{Conclusion}

The importance of an adequate dental health evaluation of medical histories in dental patients is emphasized. In this paper we believe that there is a moral duty for doctors to trust patients but we must take in consideration the importance of some degree of reliance in the past medical histories collected by the patients. Some systemic disease (Diabetes) and blood borne infection (Hepatitis) disease should be taken in consideration and accurate diagnosis and maximum of infection control must be applied in all patients to achieve high standard protection for the patients and the health care professional.

\section{Conflict of Interest}

All authors declare that no any conflict of interest exists.

\section{Bibliography}

1. Yamalik N and Van Dijk W. "Analysis of the attitudes and needs/demands of dental practitioners in the field of patient safety and risk management". International Dental Journal 63 (2013): 291-297.

2. Straus SE., et al. "Clinical assessment of the reliability of the examination (CARE)". BMJ Evidence-Based Medicine 5 (2000): 132-134.
3. Ha JF and Longnecker N. "Doctor-patient communication: a review". Ochsner Journal 10.1 (2010): 38-43.

4. Scully C and Boyle P. "Reliability of a self-administered questionnaire for screening for medical problems in dentistry". Community Dentistry and Oral Epidemiology 11 (1983): 105108.

5. Pecoraro Roger E., et al. "Validity and Reliability of a Self-Administered Health History Questionnaire". Public Health Reports 94.3 (1979): 231-238.

6. Charbonneau A., et al. "Do people with HIV/AIDS disclose their HIV-positivity to dentists?" AIDS Care 11 (1999): 61-70.

7. Caterinicchio RP. "Testing plausible path models of interpersonal trust in patient-physician treatment relationships. Social Science and Medicine". Part A: Medical Psychology and Medical Sociology 13 (1979): 81-99.

8. Rasiah S., et al. "A study of the nature and level of trust between patients and healthcare providers, its dimensions and determinants: a scoping review protocol". BMJ Open 10 (2020): e028061.

9. MacMahon B and Pugh TF. "Epideraiology: Principles and Methods". Boston, Little, Brown, and Co (1970): 261.

10. Neugut AI and Neugut RH. "How accurate are patient histories?" Journal of Community Health 9.4 (1984): 294-301.

11. Alavian SM., et al. "Survey of the level of anti-HBs antibody titer in vaccinated Iranian general dentists". Special Care in Dentistry 28.6 (2008): 265-270.

12. Saudi Ministry of Health. A Review of Health Situation. The annual health statistics book (2009).

13. Kwon SY and Lee CH. "Epidemiology and prevention of hepatitis B virus infection". The Korean Journal of Hepatology 17.2 (2011): 87-95.

14. Rustage KJ., et al. "Evaluation of a dedicated dental procedure glove for clinical dentistry". British Dental Journal 103 (1987): 193-195.

15. Crawford TJ. "State of the art practical infection control in dentistry". Journal of American Dental Association 110 (1985): 629-633.

16. Saquib S., et al. "Exploring the Knowledge, Attitude and Practice Regarding Hepatitis B Infection Among Dental Students in Saudi Arabia: A Cross-Sectional Study". Open Access Macedonian Journal of Medical Sciences 7.5 (2019): 805-809. 
17. Robert AA., et al. "Type 1 Diabetes Mellitus in Saudi Arabia: A Soaring Epidemic". International Journal of Pediatrics (2018): 9408370.

18. World Health Organization (WHO). Global report on diabetes. World Health Organization (2016).

19. Martin L M., et al. "Validation of self-reported chronic conditions and health services in a managed care population". American Journal of Preventive Medicine 18 (2000): 215-218.

20. Krajden M., et al. "The laboratory diagnosis of hepatitis B virus". Canadian Journal of Infectious Diseases and Medical Microbiology 16.2 (2005): 65-72.

21. Jargalsaikhan G., et al. "Sensitivity and specificity of commercially available rapid diagnostic tests for viral hepatitis B and C screening in serum samples". PLoS ONE 15.7 (2020): e0235036.

22. Kermani SK., et al. "Accuracy and Precision of Measured Blood Sugar Values by Three Glucometers Compared to the Standard Technique". Journal of Clinical and Diagnostic Research 11.4 (2017): OC05-0C08.

23. Eyth E., et al. “Glucose Tolerance Test (2021).

24. McKenna MT., et al. "Agreement between patient self-reports and medical records for Pap smear histories". American Journal of Preventive Medicine 8 (1992): 287-291.

25. Khalighi HR., et al. "Screening for Hepatitis B Knowledge Among Dental Patients: How Much Do they Know?" Global Journal of Dermatology and Venereology 1.1 (2013): 11-14.

26. Seitz T., et al. "Competency in medical history taking-the training physicians' view". Wiener klinische Wochenschrift 131 (2019): 17-22.

27. Olansky L and Kennedy L. "Finger-stick glucose monitoring: issues of accuracy and specificity". Diabetes Care 33.4 (2010): 948-949.

28. Handra S., et al. "Trust and Communication in a Doctor-Patient Relationship: A Literature Review". Journal of Communication in Healthcare 3 (2018): 36.

\section{Volume 5 Issue 9 September 2021}

(C) All rights are reserved by Mohamed Yaser

Kharma., et al.

Citation: Mohamed Yaser Kharma., et al. "How Accurate are Patient Histories? Hepatitis B Knowledge and Prevalence among Dental Patients". Acta Scientific Dental Sciences 5.9 (2021): 89-94. 